\title{
Topology-Aware Space-Time Network Coding
}

\author{
Rodolfo Torrea-Duran ${ }^{1}$, Máximo Morales-Céspedes ${ }^{2}$, \\ Jorge Plata-Chaves ${ }^{1}$, Luc Vandendorpe ${ }^{2}$, and Marc Moonen ${ }^{1}$ \\ ${ }^{1}$ STADIUS Center for Dynamical Systems, Signal Processing and Data Analytics \\ KU Leuven, Department of Electrical Engineering (ESAT), Leuven, Belgium \\ 2 ICTEAM Institute, Digital Communications Group, Université Catholique de Louvain (UCL), LLN, Belgium \\ \{Rodolfo.TorreaDuran, Jorge.PlataChaves, Marc.Moonen\}@esat.kuleuven.be, \{Luc.Vandendorpe, Maximo.Morales\}@uclouvain.be
}

\begin{abstract}
Space-Time Network Coding (STNC) is a timedivision multiple access (TDMA)-based scheme that combines network coding and space-time coding by allowing relays to combine the information received from different sources during the transmission phase and to forward the combined signal to a destination in the relaying phase. However, STNC schemes require all the relays to overhear the signal transmitted from all the sources in the network and also a large number of time-slots to achieve full diversity in a multipointto-multipoint transmission, which is particularly challenging for large cellular networks. In this paper, we exploit a basic knowledge of the network topology, i.e. the knowledge of the base stations overheard by other base stations and users, to reduce drastically the number of time-slots. Our results show that our scheme is able to increase the spectral efficiency with a marginal decrease of the spatial diversity compared to traditional STNC.

Index Terms-Space-time network coding, network topology, cooperative communications, relaying.
\end{abstract}

\section{INTRODUCTION}

Cooperative communication protocols exploit the broadcast nature of the wireless channel by allowing relays to retransmit the overheard information to other nodes. This is usually done in two phases. In the first phase, the source nodes broadcast the information, which is received by the destination nodes and the relays. In the second phase, the relays forward this information to the destination nodes, which combine it with the information received in the first phase. This cooperation however requires perfect timing and frequency synchronization of the received signals to avoid inter-symbol interference. The most commonly-used technique to completely avoid the imperfect synchronization issue in multi-node systems is time-division multiple access (TDMA), in which every transmission is done in a dedicated time-slot.

Space-Time Network Coding (STNC) [1] has been proposed as a TDMA-based technique to achieve cooperation among the nodes in a network while avoiding the synchronization issues. It combines network coding and space-time

This research work was carried out at the ESAT Laboratory of KU Leuven, in the frame of KU Leuven Research Council PFV/10/002 (OPTEC), FWO project G091213N "Cross-layer optimization with real-time adaptive dynamic spectrum management for fourth generation broadband access networks", and the Belgian Programme on Interuniversity Attraction Poles initiated by the Belgian Federal Science Policy Office "Belgian network on stochastic modelling, analysis, design and optimization of communication systems (BESTCOM)" 2012-2017.

ISBN 978-0-9928626-7-1 @ EURASIP 2017 coding by allowing signals coming from different source nodes during the transmission phase to be combined at the relays and then to be forwarded in dedicated time-slots during the relaying phase. Considering a system with $L$ source nodes, $M$ relays and 1 destination node, STNC is able to achieve full diversity order of $M+1$ with $L+M$ time-slots. This multipoint-to-point (M2P) transmission can be translated into a point-to-multipoint ( $\mathrm{P} 2 \mathrm{M})$ transmission by instead considering a single source node transmitting to multiple destination nodes. A multipoint-to-multipoint (M2M) transmission can be obtained by combining M2P and P2M schemes. A step towards increasing the capacity of the previous approach was taken in [2] and in [3], where the authors propose that each relay decodes the transmission not only from the source nodes but also from the previous relays. Furthermore, the source nodes can also be used as relays, avoiding in this way the deployment of relays, as proposed in clustering-based STNC [4] and optimal node selection-based STNC schemes [5].

However, to achieve such diversity order, the previous schemes require that all the $M$ relays (or the $L$ source nodes acting as relays) overhear the signal transmitted by the $L$ source nodes, which is infeasible for large networks. In a cellular downlink transmission for instance, base stations (BSs) and users typically overhear only the closest BSs and treat the other transmissions as noise [6].

Considering the previous overhearing scenario where the BSs act as relays, in this paper we propose to exploit the topology of the network by allowing simultaneous transmissions of all the BSs that do not overhear each other in the transmission phase. The overheard information is then forwarded in a single time-slot during the relaying phase. Both steps drastically reduce the number of required timeslots, however resulting in imperfect synchronization during the single time-slot of the relaying phase. Nevertheless, recent advances in delay-tolerant codes [7] or joint frequency and timing synchronization [8] show that these issues can be mitigated. The proposed scheme is analyzed here in terms of spectral efficiency and bit error rate (BER). Our results show that our scheme is able to increase the spectral efficiency compared to traditional STNC, with a small degradation in the BER. Furthermore, we compute closed-form expressions of the spectral efficiency and BER for any number of nodes, which allows us to analyze and predict the achievable gains 136 


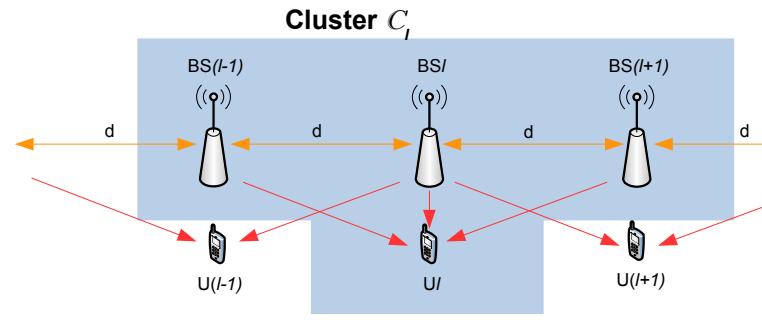

Fig. 1. Wyner model for a cellular network. The cluster considers the $2 K$ closest BSs, i.e. $\mathcal{C}_{l}=\operatorname{BS}\{l-1, l, l+1\}$ for $K=1$, with a distance $d$ between BSs.

\section{SySTEM MODEL}

We consider a downlink cellular system with $L$ singleantenna BSs, which also act as relays, and each of which has data intended for a specific user. It is assumed that the BSs and the users are half-duplex, i.e., they cannot transmit and receive simultaneously. Furthermore, there are no backhaul links among the BSs, which excludes the use of transmission schemes that require cooperation or data sharing among the BSs. Since we consider single-antenna BSs, notice that this condition excludes the use of space-time block codes, e.g., Alamouti codes. It is assumed that intracell interference is avoided through orthogonal approaches, e.g., orthogonal frequency division multiple access (OFDMA), so that we only consider the intercell interference. In this context, the user connected to the $l$-th BS, which is referred to as $\mathrm{U} l$, is only subject to intercell interference from the neighboring BSs. Specifically, we consider a topological approach where $\mathrm{U} l$ receives all the signals from the cluster $\mathcal{C}_{l}$ composed by $\mathrm{BS} l$ and its neighboring $2 K$ BSs. BSl can also overhear the transmission of the other BSs inside cluster $\mathcal{C}_{l}$. The transmissions from all the other BSs is treated as noise. Finally, it is assumed that there is no other channel state information (CSI) at each BS than the network topology, i.e. the knowledge of the BSs overheard by the considered BS and its user.

For the sake of simplicity, we focus on the widely-used linear Wyner model [9] to formulate the considered system as shown in Fig. 1. We assume a symmetric scenario where the BSs of $\mathcal{C}_{l}$ correspond to the $K$ BSs on the left (numbered $l-K, \ldots, l-1$ ) and the $K$ BSs on the right (numbered $l+$ $1, \ldots, l+K)$ of BSl. The BSs of cluster $\mathcal{C}_{l}$ that transmit in time-slot $t$ are comprised in the set $\mathcal{C}_{l}^{(t)}$. Thus, assuming $\mathrm{BS} l \in \mathcal{C}_{l}^{(t)}$, the signal received by $\mathrm{U} l$ in time-slot $t$ in the transmission phase can be written as

$$
y_{l}^{(t)}=\sqrt{P_{l}} h_{l l} s_{l}+\sum_{\mathrm{BS} k \in \mathcal{C}_{l}^{(t)} \backslash \mathrm{BS} l} i_{k l}^{(t)}+n_{l}^{(t)},
$$

where $h_{k l}$ is the channel between BSk and user $\mathrm{U} l$, which follows a Rayleigh distribution with zero mean and unit variance. Furthermore, $P_{l}$ is the transmit power of $\mathrm{BS} l, s_{l}$ is the symbol transmitted by BS $l$ intended for $\mathrm{U} l$, and $n_{l}^{(t)}$ is the additive white Gaussian noise (AWGN) with zero mean ISBN 978-0-9928626-7-1 (C) EURASIP 2017 and unit variance $\sigma_{\mathrm{n}}^{2}$, which is assumed to be equal for all the users. Finally, $i_{k l}^{(t)}$ denotes the interference received by $\mathrm{U} l$ because of the transmission of $\mathrm{BS} k$ in time-slot $t$. Therefore, $\sum_{\mathrm{BS} k \in \mathcal{C}_{l}^{(t)} \backslash \mathrm{BS} l} i_{k l}^{(t)}$ corresponds to the intercell interference within the cluster of interest. The signal-tonoise ratio (SNR) of $\mathrm{U} l$ received from $\mathrm{BS} k$ can be defined as

$$
\gamma_{k l}=\frac{P_{k}\left|h_{k l}\right|^{2} \sigma_{\mathrm{s}}^{2}}{\sigma_{\mathrm{n}}^{2}}
$$

where $\sigma_{\mathrm{s}}^{2}=\mathbb{E}\left\{\left|s_{l}\right|^{2}\right\} \forall l$.

\section{BASELINE SCHEMES}

This section presents the baseline schemes which are used to benchmark the performance of the proposed scheme.

\section{A. Simultaneous transmissions scheme}

Assume that all the BSs transmit simultaneously in each time-slot regardless of the interference that they cause to the other users. This means that the transmission phase has one time-slot $(t=1)$ and that there is no relaying phase. We refer to this approach as INTF. The signal received by $\mathrm{U} l$ is given by (1). Thus, the spectral efficiency per time-slot for $\mathrm{U} l$ in time-slot $t=1$ with $\mathcal{C}_{l}^{(t)}=\mathcal{C}_{l}$ can be directly computed as

$$
S_{l}^{\mathrm{INTF}}=\mathbb{E}\left\{\log _{2}\left(1+\frac{\gamma_{l l}}{1+\sum_{\substack{k=l-K \\ k \neq l}}^{l+K} \gamma_{k l}}\right)\right\} .
$$

Note that the full multiplexing gain due to the use of every available time-slot $(2 K+1$ symbols are transmitted simultaneously) corresponds to a considerable degradation in equivalent signal-to-interference-plus-noise ratio (SINR) of each user.

For BPSK modulation over a Rayleigh fading channel, the BER can be computed in terms of the $Q$ function as

$$
\mathrm{BER}_{l}^{\mathrm{INTF}}=\mathbb{E}\left\{Q\left(\sqrt{2 \gamma_{\mathrm{INTF}}}\right)\right\},
$$

where $\gamma_{\mathrm{INTF}}=\gamma_{l l} /\left(1+\sum_{\substack{k=l-K \\ k \neq l}}^{l+K} \gamma_{k l}\right)$.

\section{B. Modified STNC (mSTNC)}

In a scenario where each BSs overhears its neighboring $2 K$ BSs, achieving full diversity order requires dedicated time-slots per transmission so that each user receives its desired symbol from $2 K+1$ signal paths. For this, we redefine the M2M transmission scheme using STNC [1] and we refer to this approach mSTNC.

In a first phase each BS of the cluster transmits the symbol intended for its corresponding user in a dedicated time-slot while all the other BSs remain silent. In a second phase, each BS of the cluster transmits one of the symbols overheard in 
TABLE I

MSTNC TRANSMISSION STRATEGY $(K=1)$

\begin{tabular}{|c|c|c|c|c|c|}
\hline & $\mathrm{BS}(l-2)$ & $\mathrm{BS}(l-1)$ & $\mathrm{BS} l$ & $\mathrm{BS}(l+1)$ & $\mathrm{BS}(l+2)$ \\
\hline TS1 & & $s_{l-1}$ & & & $s_{l+2}$ \\
\hline TS2 & & & $s_{l}$ & & \\
\hline TS3 & $s_{l-2}$ & & & $s_{l+1}$ & \\
\hline TS4 & & $s_{l}$ & & & $s_{l+3}$ \\
\hline TS5 & & & $s_{l+1}$ & & \\
\hline TS6 & $s_{l-1}$ & & & $s_{l+2}$ & \\
\hline TS7 & & $s_{l-2}$ & & & $s_{l+1}$ \\
\hline TS8 & & & $s_{l-1}$ & & \\
\hline TS9 & $s_{l-3}$ & & & $s_{l}$ & \\
\hline
\end{tabular}

the first phase in a dedicated time-slot. In a third phase, each BS of the cluster transmits another of the symbols overheard in the first phase in a dedicated time-slot. This process continues until in phase $2 K+1$ each BS of the cluster transmits the last of the symbols overheard in the first phase in a dedicated time-slot. Hence this approach requires $(2 K+1)^{2}$ time-slots.

The transmission strategy of mSTNC for $K=1$ is summarized in Table I. Focusing on the $l$-th user for the case $K=1$, the desired symbol $s_{l}$ is received in three time-slots corresponding to the transmission from BSs $\{l-1, l, l+1\}$. The received signal can then be expressed as

$$
\begin{aligned}
& y_{l}^{(2)}=\sqrt{P_{l}} h_{l l} s_{l}+n_{l}^{(2)} \\
& y_{l}^{(4)}=\sqrt{P_{l-1}} h_{l-1, l} z_{l, l-1}^{(2)}+n_{l}^{(4)} \\
& y_{l}^{(9)}=\sqrt{P_{l+1}} h_{l+1, l} z_{l, l+1}^{(2)}+n_{l}^{(9)}
\end{aligned}
$$

with $z_{m n}^{(t)}=s_{m}+\frac{n_{\mathrm{BS} n}^{(t)}}{\sqrt{P_{m}} g_{m n}}$, where $g_{m n}$ is the channel between $\mathrm{BS} m$ and $\mathrm{BS} n$ and $n_{\mathrm{BS} n}^{(t)}$ is the AWGN noise received by $\mathrm{BS} n$ in time-slot $t$. Notice that the interference coming from the BSs inside the cluster is completely avoided.

The spectral efficiency per time-slot of mSTNC for $\mathrm{U} l$ (using an MMSE receiver as described in Section IV) can be directly computed for the general case $K \geq 1$ as

$$
S_{l}^{\mathrm{mSTNC}}=\frac{1}{(2 K+1)^{2}} \mathbb{E}\left\{\log _{2}\left(1+\gamma_{\mathrm{mSTNC}}\right)\right\},
$$

where

$$
\gamma_{\mathrm{mSTNC}}=\gamma_{l l}+\sum_{\substack{k=l-K \\ k \neq l}}^{l+K} \frac{\gamma_{k l}}{\frac{\gamma_{k l}}{\xi_{l k}}+1}
$$

and $\xi_{m n}=\frac{P_{m}\left|g_{m n}\right|^{2} \sigma_{\mathrm{s}}^{2}}{\sigma_{\mathrm{n}}^{2}}$. Notice that the spectral efficiency has a pre-log factor of $\frac{1}{(2 K+1)^{2}}$, which can be interpreted as the multiplexing gain. In contrast to the simultaneous transmissions scheme, it can be seen that the diversity achieved from the transmission of different BSs involves a considerable penalty in multiplexing gain.

The BER of $\mathrm{U} l$ can be directly computed as follows

$$
\mathrm{BER}_{l}^{\mathrm{mSTNC}}=\mathbb{E}\left\{Q\left(\sqrt{2 \gamma_{\mathrm{mSTNC}}}\right)\right\} .
$$

Since $\gamma_{\mathrm{mSTNC}}$ is not affected by the interference from other BSs within the cluster, mSTNC can achieve full diversity order of $2 K+1$.
TABLE II

TAS TRANSMISSION STRATEGY $(K=1)$

\begin{tabular}{|c|c|c|c|c|c|}
\hline & $\mathrm{BS}(l-2)$ & $\mathrm{BS}(l-1)$ & $\mathrm{BS} l$ & $\mathrm{BS}(l+1)$ & $\mathrm{BS}(l+2)$ \\
\hline TS1 & & $s_{l-1}$ & & $s_{l+1}$ & \\
\hline TS2 & $s_{l-2}$ & & $s_{l}$ & & $s_{l+2}$ \\
\hline & $s_{l-3}+$ & $s_{l-2}+$ & $s_{l-1}+$ & $s_{l}+$ & $s_{l+1}+$ \\
TS3 & $s_{l-1}$ & $s_{l}$ & $s_{l+1}$ & $s_{l+2}$ & $s_{l+3}$ \\
\hline \hline
\end{tabular}

\section{TOPOLOGY-AwARE STNC}

The previous schemes determine the achievable spectral efficiency and BER for extreme approaches such as maximizing the diversity or maximizing the multiplexing gain. Here we exploit a basic knowledge of the network topology to find a trade-off between the number of time-slots and the diversity order.

During the first time-slot of the transmission phase a pair of BSs within the cluster of interest that do not overhear each other, i.e. $\mathrm{BS}(l-K)$ and $\mathrm{BS}(l+1)$, transmits simultaneously the symbol intended for their corresponding user. In the next time-slot the next pair, i.e. BS $(l-K+1)$ and $\mathrm{BS}(l+2)$, transmits. Finally, in the $(K+1)$-th timeslot the middle BS of the cluster, i.e. BSl, transmits its symbol. Meanwhile, the BSs out of the cluster of interest reuse the transmission resources by using a similar transmission strategy. Once the transmission phase concludes, each BS has overheard a combination of the symbols from its $2 K$ neighboring BSs. Then, in the relaying phase the BSs transmit simultaneously the combination of overheard symbols in a single time-slot.

The transmission strategy of the proposed approach for $K=1$ is summarized in Table II and we refer to it as Topology-Aware STNC (TAS). In this case, the received signals for $\mathrm{U} l$ can be expressed as

$$
\begin{aligned}
& y_{l}^{(1)}=\sqrt{P_{l-1}} h_{l-1, l} s_{l-1}+\sqrt{P_{l+1}} h_{l+1, l} s_{l+1}+n_{l}^{(1)} \\
& y_{l}^{(2)}=\sqrt{P_{l}} h_{l, l} s_{l}+n_{l}^{(2)} \\
& y_{l}^{(3)}=\sum_{\substack{k=l-1 \\
k \neq l}}^{l+1} \sqrt{P_{k}} h_{k, l} \psi_{k}^{(1)}+\sqrt{P_{l}} h_{l, l} \psi_{l}^{(2)}+n_{l}^{(3)},
\end{aligned}
$$

where

$\psi_{k}^{(t)}=$

$\frac{s_{k-1}}{\sqrt{P_{k+1}} g_{k+1, k}}+\frac{s_{k+1}}{\sqrt{P_{k-1}} g_{k-1, k}}+\frac{n_{\mathrm{BS} k}^{(t)}}{\sqrt{P_{k-1}} g_{k-1, k} \sqrt{P_{k+1}} g_{k+1, k}}$.

It is worth to mention that a normalization in (10) is necessary to avoid exceeding the maximum power with which the BSs can transmit.

In the general case $K \geq 1$, the signal received by user $\mathrm{U} l, \mathbf{y}_{l} \in \mathbb{C}^{(K+2) \times 1}$, for the proposed scheme can be written as equation (11) where $\operatorname{diag}(\mathbf{a})$ denotes the diagonal matrix of the vector $\mathbf{a}, \mathbf{s}_{\text {cluster }}=\operatorname{col}\left\{s_{l^{\prime}}\right\}_{l^{\prime}=l-K}^{l+K}$ are the symbols intended for the users within the cluster and $\mathbf{s}_{\mathrm{left}}=\operatorname{col}\left\{s_{l^{\prime}}\right\}_{l^{\prime}=l-2 K}^{l-K-1}$ and $\mathbf{s}_{\text {right }}=\operatorname{col}\left\{s_{l^{\prime}}\right\}_{l^{\prime}=l+K+1}^{l+2 K}$ $138^{\text {ate }}$ 


$$
\mathbf{y}_{l}=\underbrace{\left[\begin{array}{ccccc}
\mathbf{0}_{K, K} & \operatorname{diag}\left(\left\{\sqrt{\gamma_{l^{\prime}, l}}\right\}_{l^{\prime}=l-1}^{l-K}\right) & \mathbf{0}_{K, 1} & \operatorname{diag}\left(\left\{\sqrt{\gamma_{l^{\prime}, l}}\right\}_{l^{\prime}=l+1}^{l+K}\right) & \mathbf{0}_{K, K} \\
\mathbf{0}_{1, K} & \mathbf{0}_{1, K} & \begin{array}{c}
\sqrt{\gamma_{l l}} \\
\mathbf{h}_{\text {cluster }}
\end{array} & \mathbf{0}_{1, K} & \mathbf{0}_{1, K} \\
\mathbf{h}_{\text {left }} & & & \mathbf{h}_{\text {right }}
\end{array}\right]}_{\mathbf{A}}\left[\begin{array}{c}
\mathbf{s}_{\text {left }} \\
\mathbf{s}_{\text {cluster }} \\
\mathbf{s}_{\text {right }}
\end{array}\right]+\mathbf{n}_{l},
$$

the left- and right-hand side, respectively. Moreover,

$\mathbf{h}_{\text {cluster }}=\left[\begin{array}{lllllll}\eta_{l-K, l} & \ldots & \eta_{l-1, l} & \eta_{l, l} & \eta_{l+1, l} & \ldots & \eta_{l+K, l}\end{array}\right]$

shows the channel entries for the symbols $\mathbf{s}_{\text {cluster, }}$ where we define

$$
\eta_{m l}=\sum_{l^{\prime}=m-K}^{m-1} \frac{\sqrt{\gamma l^{\prime}, l}}{\sqrt{\xi_{m-K-1, l^{\prime}}}}+\sum_{l^{\prime}=m+1}^{m+K} \frac{\sqrt{\gamma_{l^{\prime}, l}}}{\sqrt{\xi_{m+K+1, l^{\prime}}}} .
$$

Similarly, $\mathbf{h}_{\text {left }}=\left[\begin{array}{llll}\eta_{l-2 K, l} & \ldots & \eta_{l-K-1, l}\end{array}\right]$ and $\mathbf{h}_{\text {right }}=$ $\left[\begin{array}{lll}\eta_{l+K+1, l} & \ldots & \eta_{l+2 K, l}\end{array}\right]$ are the channel entries for the symbols $\mathbf{s}_{\text {left }}$ and $\mathbf{s}_{\text {right }}$, respectively, and $\mathbf{n}_{l}$ contains the noise and interference from the BSs outside of the cluster

$\mathbf{n}_{l}=$

$\left[n_{l}^{(1)} \quad \cdots \quad n_{l}^{(K+1)}\left(n_{l}^{(K+2)}+\sum_{\substack{k=l-K \\ t=\{1, \ldots, K+1\}}}^{l+K} \sqrt{P_{k}} h_{k l} \phi_{k}^{(t)}\right)\right]^{T}$

where

$$
\phi_{k}^{(t)}=\frac{n_{\mathrm{BS} k}^{(t)}}{\sqrt{P_{k-1}} g_{k-1, k} \sqrt{P_{k+1}} g_{k+1, k}}
$$

is the forwarded noise.

From (11), the received signal of $\mathrm{U} l$ is given in vector form as

$$
\mathbf{y}_{l}=\sum_{k=l-2 K}^{l+2 K} \mathbf{a}_{k} s_{k}+\mathbf{n}_{l}=\mathbf{a}_{l} s_{l}+\mathbf{w}_{l}
$$

where $\mathbf{a}_{l}$ is the $l$-th column of matrix $\mathbf{A}$ and $\mathbf{w}_{l}=$ $\sum_{\substack{k=l-2 K \\ k \neq l}}^{l+2 K} \mathbf{a}_{k} s_{k}+\mathbf{n}_{l}$.

The MMSE receiver [11] can be derived by whitening the colored-noise term $\mathbf{w}_{l}$ with its covariance matrix $\mathbf{R}_{w_{l}}$ and then taking the inner product of the remaining signal and the vector $\mathbf{R}_{w_{l}}^{-1 / 2} \mathbf{a}_{l}$. The SNR of $\mathrm{U} l$ using the MMSE receiver can be shown to be

$$
\mathrm{SNR}_{l}^{\mathrm{TAS}}=\frac{\mathbb{E}\left\{\left|\hat{z}_{\mathrm{sig}}\right|^{2}\right\}}{\mathbb{E}\left\{\left|\hat{z}_{\text {noise }}\right|^{2}\right\}}=\mathbf{a}_{l}^{H} \mathbf{R}_{w_{l}}^{-1} \mathbf{a}_{l} \sigma_{\mathrm{s}}^{2} .
$$

The spectral efficiency per time-slot of $\mathrm{U} l$ is then

$$
S_{l}^{\mathrm{TAS}}=\frac{1}{K+2} \log _{2}\left(1+\mathbf{a}_{l}^{H} \mathbf{R}_{w_{l}}^{-1} \mathbf{a}_{l} \sigma_{\mathrm{s}}^{2}\right) .
$$

The $\frac{1}{K+2}$ scaling factor corresponds to the multiplexing gain due to the number of time-slots used for each transmission ISBN 978-0-9928626-7-1 @ EURASIP 2017 round. Moreover, $\mathbf{R}_{w_{l}}$ is given as

$$
\mathbf{R}_{w_{l}}=\sum_{\substack{k=l-2 K \\ k \neq l}}^{l+2 K} \mathbf{a}_{k} \mathbf{a}_{k}^{H} \sigma_{\mathrm{s}}^{2}+\mathbf{N}
$$

where $\mathbf{N}=\operatorname{diag}\left[\begin{array}{llll}\sigma_{\mathrm{n}}^{2} & \sigma_{\mathrm{n}}^{2} & \cdots & \sigma_{\mathrm{n}}^{2}+\sigma_{\phi_{k}^{(K+2)}}^{2}\end{array}\right]^{T}$ and $\sigma_{\phi_{k}^{(t)}}^{2}$ is the power of the forwarded noise in time-slot $t$.

It can then be shown that $S_{l}^{\mathrm{TAS}}$ results in equation (20) where $\hat{\eta}_{m l}$ is defined as $\eta_{m l}$ but with $l^{\prime} \in \mathcal{C}_{l}$ and

$\Psi_{m l}=$

$\frac{\left(\sqrt{\gamma_{m l}} \hat{\eta}_{m+K+1, l}-\sqrt{\gamma_{m+K+1, l}} \hat{\eta}_{m l}\right)^{2}+N_{m}\left(\hat{\eta}_{m l}^{2}+\hat{\eta}_{m+K+1, l}^{2}\right)}{\gamma_{m l}+\gamma_{m+K+1, l}+N_{m}}$

It can be seen that the term $\gamma_{l l}$ in equation (20) corresponds to the SNR coming from BSl. On the other hand the term $\Delta$ corresponds to the contribution of the other BSs in the cluster. Specifically, $\eta_{l l}^{2}$ is the received power from all the BSs inside the cluster except $\mathrm{BSl}$ and it is divided by the noise power $\sigma_{\mathrm{n}}^{2}$, by the received power from the symbols of the cluster coming from the BSs outside the cluster $\sum_{\substack{m=l-2 K \\ m \notin \mathcal{C}}}^{l+2 K} \hat{\eta}_{m l}^{2}$, and by $\sum_{\substack{m=l-K \\ m \neq l}}^{l} \Psi_{m l}$, which represents a penalty from the simultaneous transmissions of $K$ BSs inside the cluster.

The BER of $\mathrm{U} l$ can be calculated as

$$
\mathrm{BER}_{l}^{\mathrm{TAS}}=\mathbb{E}\left\{Q\left(\sqrt{2\left(\gamma_{l l}+\Delta\right)}\right)\right\} .
$$

It can be seen that this scheme allows simultaneous transmissions while still achieving diversity from multiple BSs.

\section{Performance Evaluation}

In this section we compare the performance of the proposed TAS scheme with the baseline schemes described in Section III. We consider the Wyner model of Fig. 1. For simplicity, we assume equal transmit power during each time-slot and each BS, i.e. $P_{1}=P_{2}=P_{3}=\cdots$. Our evaluations consider a Rayleigh fading channel model with $\mathbb{E}\left\{\left|h_{m l}\right|^{2}\right\}=\mathbb{E}\left\{\left|g_{m n}\right|^{2}\right\}=1 \forall m, l, n$ and $\sigma_{\mathrm{s}}^{2}=\sigma_{\mathrm{n}}^{2}=1$.

The spectral efficiency per time-slot of the studied schemes as a function of the equivalent SNR to the singleBS is depicted in Fig. 2 for $K=1$ and $K=4$. It can be seen that the TAS scheme outperforms INTF and mSTNC schemes as the SNR increases. Notice that the spectral efficiency for the mSTNC scheme suffers a large penalty in the pre-log factor due to the number of time-slots required. On 


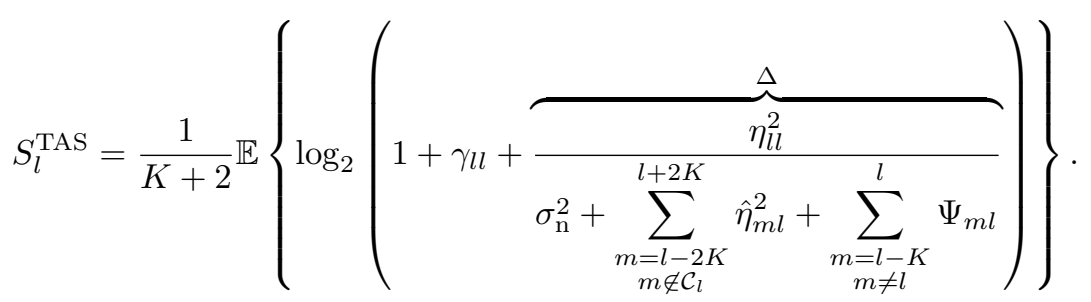

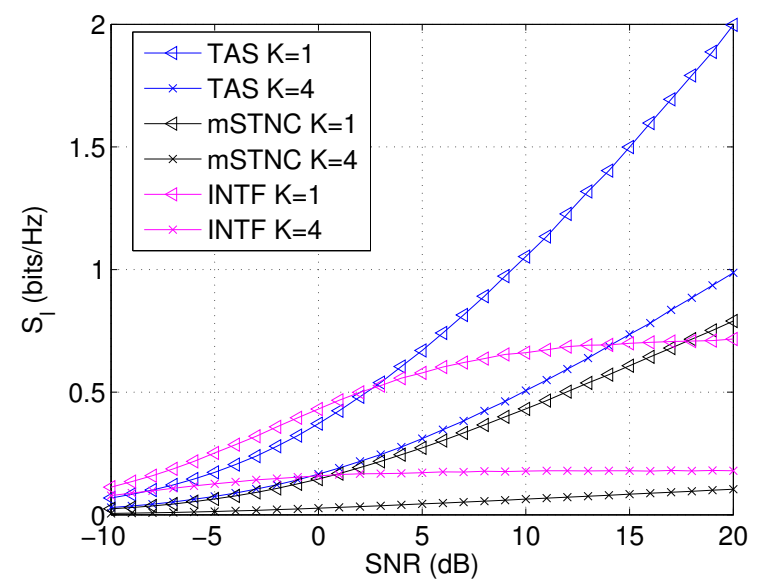

Fig. 2. Spectral efficiency per time-slot of the studied schemes with $K=1$ and $K=4$.

the other hand, INTF achieves a low performance because of the received interference that increases with the SNR.

The BER performance of the studied schemes is depicted in Fig. 3. It can be seen that INTF and mSTNC correspond to the upper and lower bounds of the BER, respectively. At this point it is interesting to recall that mSTNC obtains a low BER and full diversity order at the cost of a large number of time-slots. However, the proposed TAS approach achieves a BER higher than mSTNC, but much lower than INTF. Therefore, by taking advantage of the network topology, the proposed TAS scheme achieves a performance that provides a trade-off between multiplexing gain and diversity, outperforming in this way the baseline schemes.

\section{CONCLUSIONS}

In this paper we have proposed a topology-aware STNC scheme that exploits a basic knowledge of the network topology, i.e. the knowledge of the BSs that can be overheard by other BSs and users, using the Wyner cellular model. This allows to perform simultaneous transmissions of the BSs that do not overhear each other during the transmission phase and simultaneous transmissions of all the BSs during the relaying phase. Our results show a dramatic increase in the spectral efficiency per time-slot compared to traditional STNC due to the increase in multiplexing gain with a small degradation in the BER.

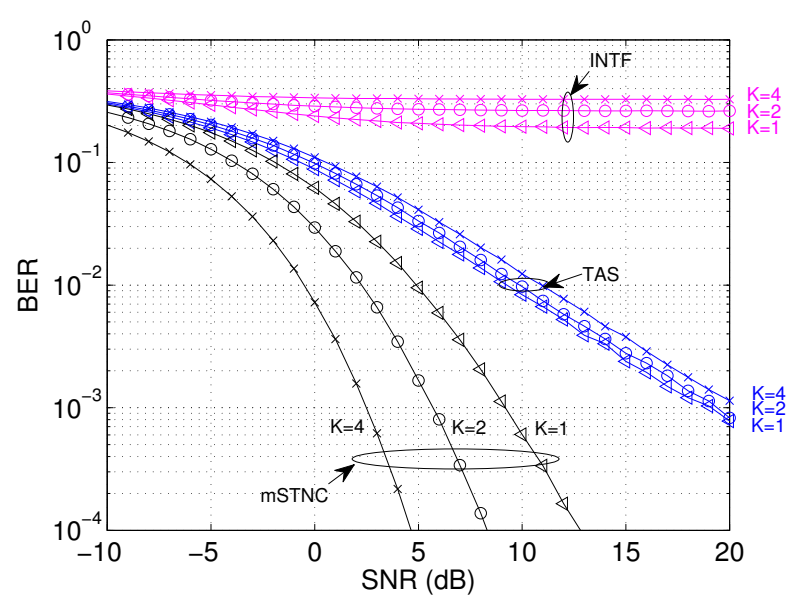

Fig. 3. BER of the studied schemes.

\section{REFERENCES}

[1] H. Q. Lai, and K. J. R. Liu, "Space Time Network Coding", IEEE Transactions on Signal Processing, vol. 59, no. 4, Apr. 2011.

[2] K. Xiong, P. Fan, H. C. Yang,and K. B. Letaief, "Space Time Network Coding with Overhearing Relays", IEEE Transactions on Signal Processing, vol. 13, no. 7, Jul. 2014.

[3] Y. Zhang, K. Xiong, P. Fan, X. Di, and X. Zhou, "Outage Performance of Space Time Network Coding with Overhearing AF Relays", IEEE Communications Letters, vol. 19, no. 12, Dec. 2015.

[4] W. Guan and K. J. Rai Liu, "Clustering Based Space-Time Network Coding" GLOBECOM, Dec. 2012, pp. 5633-5638.

[5] M. W. Baidas and A. B. MacKenzie, Many-to-many spacetime network coding for amplify-and-forward cooperative networks: Node selection and performance analysis, EURASIP Journal on Wireless Communications and Networking, pp. 117, 2014.

[6] C. Geng, N. Naderializadeh, A. S. Avestimehr and S. A. Jafar, "On the Optimality of Treating Interference as Noise", IEEE Transactions on Information Theory, vol. 61, no. 4, Dec. 2015.

[7] M. Damen and A. Hammons, Delay-tolerent distributed-TAST codes for cooperative diversity, IEEE Transactioins on Information Theory, vol. 53, no. 10, pp. 2941-2956, Oct. 2007.

[8] J. Zhang, C. Shen, G. Deng, and Y. Wang, Timing and Frequency Synchronization for Cooperative Relay Networks, Vehicular Technology Conference (VTC), Sep. 2013.

[9] J. Xu, J. Zhang, and J. G. Andrews, "On the Accuracy of the Wyner Model in Cellular Networks", IEEE Transactions on Wireless Communications, vol. 10, no. 0, Sep. 2011.

[10] A. Goldsmith, "Wireless Communication", Cambridge University Press, 2005.

[11] D. Tse and P. Viswanath, "Fundamentals of Wireless Communications", Cambridge University Press, 2005. 